Introduction Palliative sedation is sometimes used to manage refractory symptoms in adult palliative care patients in the UK. However, there is limited knowledge regarding which observational tools are most appropriate for monitoring the effects of sedative drugs. This review is a part of the I-CANCARE, a Marie Curie funded research programme.

Objective To identify and critique primary studies describing the use of observational measures to assess the depth of sedation at the end of life.

Methods We searched six databases (CENTRAL, Cinahl, Embase, Medline, PsychINFO, Scopus, and Web of Science) until November 2016, using search terms combining subject headings and free-text terms. Primary studies reporting on the use of observational measures to assess the effect of palliative sedation were eligible for inclusion. Two investigators independently reviewed the titles, abstracts and full-text articles retrieved, and performed the data extraction. From the included papers, a list of observational tools, their characteristics and the degree to which they have been validated, using the COSMIN checklist (Mokkink et al., 2010), will be generated.

Results to-date The searches yielded 10208 articles after removing duplicates. The majority of studies meeting the inclusion criteria and discussing the monitoring of sedation reported using observational measures for assessing not only depth of sedation but also symptom severity. Of the identified scales assessing management of intractable symptoms, only one had been validated for palliative care settings. Five measures assessing level of consciousness had undergone partial validation for use in palliative care. Six of the observational measures identified had been evaluated for their psychometric properties in single study populations.

Conclusions Few measures for monitoring sedation have been evaluated for their psychometric properties in palliative care. Next steps will be a detailed evaluation of the overall quality of the studies identified, and of the validity and reliability of the scales used. Definitive results will become available in February 2017.

\section{P-108 EVIDENCING CARE OF THE DYING ADULT IN A DISTRICT GENERAL HOSPITAL}

Sinead Henderson, Susan Dargan, Ria Wright, Clare Smith. Ashford and St Peters NHS Trust, Chertsey, UK

\subsection{6/bmjspcare-2017-00133.107}

Background Recent national documents, such as One Chance to Get it Right and Ambitions for Palliative and End of Life Care, have highlighted the importance of high quality end of life care. In particular they have promoted the use of individualised care planning for the dying adult. Ashford and St Peters NHS Trust is a busy district general hospital in Surrey and has over 1000 deaths per year. To ensure that high quality care was delivered to all by all healthcare professionals an individualised care plan was devised. A baseline audit was undertaken before this was rolled out across the trust.

Methods An audit tool was created based on the 5 priorities of care and NICE guidance for care of the dying adult. This was to assess the documentation of care given to the dying adult and their family. A retrospective notes review of the first 100 deaths in quarter 1 was undertaken.
Results Recognition of dying on the wards ranged from 50\%$100 \%$ and was initially documented by the medical team in $60 \%$. Most patients were recognised as dying in the last 48 hours. Almost $80 \%$ of patients were unable to participate in decision making about their care, although family was involved in over 95\%. Over 55\% were prescribed anticipatory medication and over 20\% had evidence of a holistic assessment. Less than $5 \%$ of notes reviewed showed evidence of adequate individualised care planning in the last days of life.

Conclusion This audit has highlighted that health professionals were recognising dying. Late recognition leads to patients not being involved in their care planning and achieving their priorities of care. Documentation and care is reliant on individual health care professionals approach rather than an informed, standardised practice. Individualised care planning can support healthcare professionals in delivering and evidencing this care.

\section{P-109 TRANSFORMING AN ACUTE PALLIATIVE CARE SERVICE IN A SPECIALIST EMERGENCY CARE HOSPITAL}

${ }^{1}$ Katherine Frew, ${ }^{1}$ Leonie Armstrong, ${ }^{1}$ Louise Whitfield, ${ }^{2}$ Jennifer Samuelson, ${ }^{2}$ Anna Office, ${ }^{1}$ Carole Duff, ${ }^{1}$ Hannah Hall, ${ }^{2}$ Patricia Robson. ${ }^{1}$ Northumbria Healthcare NHS FT, North Shields, UK; ${ }^{2}$ Marie Curie

\subsection{6/bmjspcare-2017-00133.108}

Background Northumbria Healthcare NHS FT has developed a novel approach to providing emergency care with a new Northumbria Specialist Emergency Care Hospital (NSECH), which opened in 2015. This innovative model of care has been highlighted by NHS England as an example of good practice and, in line with the five year forward view ${ }^{1}$, may be replicated in other areas of the country. Northumbria has developed a partnership with Marie Curie which has enabled successful team expansion. It is imperative to assess the impact of such a development on existing and developing palliative care services.

Design This service evaluation compared the number of patients, number of face to face contacts, duration of episode of care, and the time from referral to first contact, before and after the opening of NSECH. Data were extracted from an existing palliative care database.

Results In 2014, 439 patients were seen, with a total of 830 face to face patient contacts.

In 2016, 873 patients were seen, with a total of 2610 face to face patient contacts.

In 2014 the mean duration of the episode of care was 18 days: in 2016 this was 10 days. In NSECH the median was 1 day; the mean was 3 days (range 0-157 days).

In $201469 \%$ of patients were seen within 24 hours of referral; in 2016 this had risen to $96 \%$.

Discussion The opening of a NSECH has transformed the model of the hospital palliative care liaison team, with a $99 \%$ increase in patients seen, $199 \%$ increase in face to face contacts with patients and a significant reduction in the duration of an episode of care. The service now incorporates a hyperacute palliative care service which has an implication for service development for all trusts considering a similar approach to care.

\section{REFERENCES}

1. http://www.england.nhs.uk/ourwork/futurenhs/ accessed on 11/11/2016. 